\title{
Telotristat Etiprate
}

National Cancer Institute

\section{Source}

National Cancer Institute. Telotristat Etiprate. NCI Thesaurus. Code C81941.

An orally bioavailable, small-molecule, tryptophan hydroxylase (TPH) inhibitor prodrug, with potential antiserotonergic activity. Upon administration, telotristat etiprate is converted to its active moiety, telotristat (LP-778902), which binds to and blocks the activity of TPH. This may result in a reduction in peripheral serotonin (5-HT) production and improvement of serotonin-mediated gastrointestinal effects such as severe diarrhea. TPH, the rate-limiting enzyme in serotonin biosynthesis, is overexpressed in carcinoid tumor cells. 